\title{
Comparative Analysis of Two Cryogenic Force Balance Calibration Systems
}

\author{
Kenneth G. Toro*, Devin Burns ${ }^{\dagger}$, and Peter A. Parker ${ }^{\ddagger}$ \\ NASA Langley Research Center, Hampton, Virginia, 23681, USA
}

\begin{abstract}
Cryogenic wind-tunnel facilities face unique challenges in the calibration and operation of various measurement systems and instrumentation. Instruments that are subjected to the cryogenic conditions of the test plenum require careful design and calibration procedures to maintain instrument performance. NASA's National Transonic Facility (NTF) and the European Transonic Windtunnel (ETW) are two cryogenic wind-tunnel facilities, each with the ability to calibrate force measurement systems (FMS) at cryogenic conditions. These facilities have different methodologies and processes for calibrating these systems. This paper discusses differences in the methodologies and processes and compares the results of two separate cryogenic calibrations of the NTF-118A force balance that were completed at both wind-tunnel facilities.
\end{abstract}

\section{Nomenclature}

BCM Balance Calibration Machine

ETW European Transonic Windtunnel

FMS Force Measurement System

LaRC Langley Research Center

NASA National Aeronautics and Space Administration

\section{Background}

NASA's National Transonic Facility (NTF) and the European Transonic Windtunnel (ETW) are two of the premier high Reynolds number, cryogenic, wind tunnels in the world. These wind-tunnel facilities operate in a temperature range between $110 \mathrm{~K}$ and $320 \mathrm{~K}$ to achieve the proper wind-tunnel conditions. ${ }^{1,2}$ For instrumentation in the test plenum to provide accurate measurements, the measurement systems need to be properly conditioned and calibrated. This includes the internal wind tunnel balances at both of these facilities, which are fully exposed to the test plenum conditions.

Entries of NASA's Common Research Model at NTF and ETW have shown different results at high Reynolds numbers and low temperatures. ${ }^{2}$ Each facility used their own model support hardware and instrumentation for the entries including their own wind tunnel balance. One suggestion for the tunnel differences was differences in the balance calibration methodologies used by NASA and ETW.

Both facilities devised unique methodologies for balance calibrations at cryogenic temperatures. NASA balance calibrations rely on gravity based loads using calibrated weights, which is the traditional method for calibrating balances. At ETW, balance calibrations are performed using an automated Balance Calibration Machine (BCM) that applies loads through load generators, while maintaining the balance at various temperatures. ${ }^{3}$

To provide a direct comparison between the two calibration methodologies, NASA's NTF 118A balance was calibrated at both NASA and ETW. This work aims to provide an in depth comparison of the room

\footnotetext{
*Research Engineer, System Engineering and Engineering Methods, NASA Langley Research Center

$\dagger$ Balance Engineer, Advanced Measurement and Data Systems, NASA Langley Research Center

$\ddagger$ Team Lead, Advanced Measurement and Data Systems, NASA Langley Research Center
} 
temperature calibrations. Particular emphasis will be on the discrepancies between the terms of the matrices and whether these differences can potentially be explained by balance calibration coordinate system errors.

\section{Calibration Comparison Analysis}

The calibration of a balance on different calibration systems is expected to yield different results. A myriad of factors contribute to observed discrepancies such as, level reference, balance fit, deflections and etc. This section will look into the discrepancies found between calibrations of the NTF-118A at ETW and NASA and some of the possible sources.

\section{A. Calibration Methodologies}

Prior to an analysis of the two calibrations, it is important to note the differences in the executions of the calibrations. Rhew and Parker discuss several aspects of the differences between ETW and NASA room temperature calibrations in terms of load points. ${ }^{4}$ One of the unique challenges with this study is the differences in the full-scale loads that were achievable by the BCM with respect to NASA. For example, the BCM was capable of apply $35 \%$ of the full-scale load for side force of the NTF-118A. ${ }^{5}$ This is not a particular drawback of the system, since its capability was intended for ETW's load ranges.

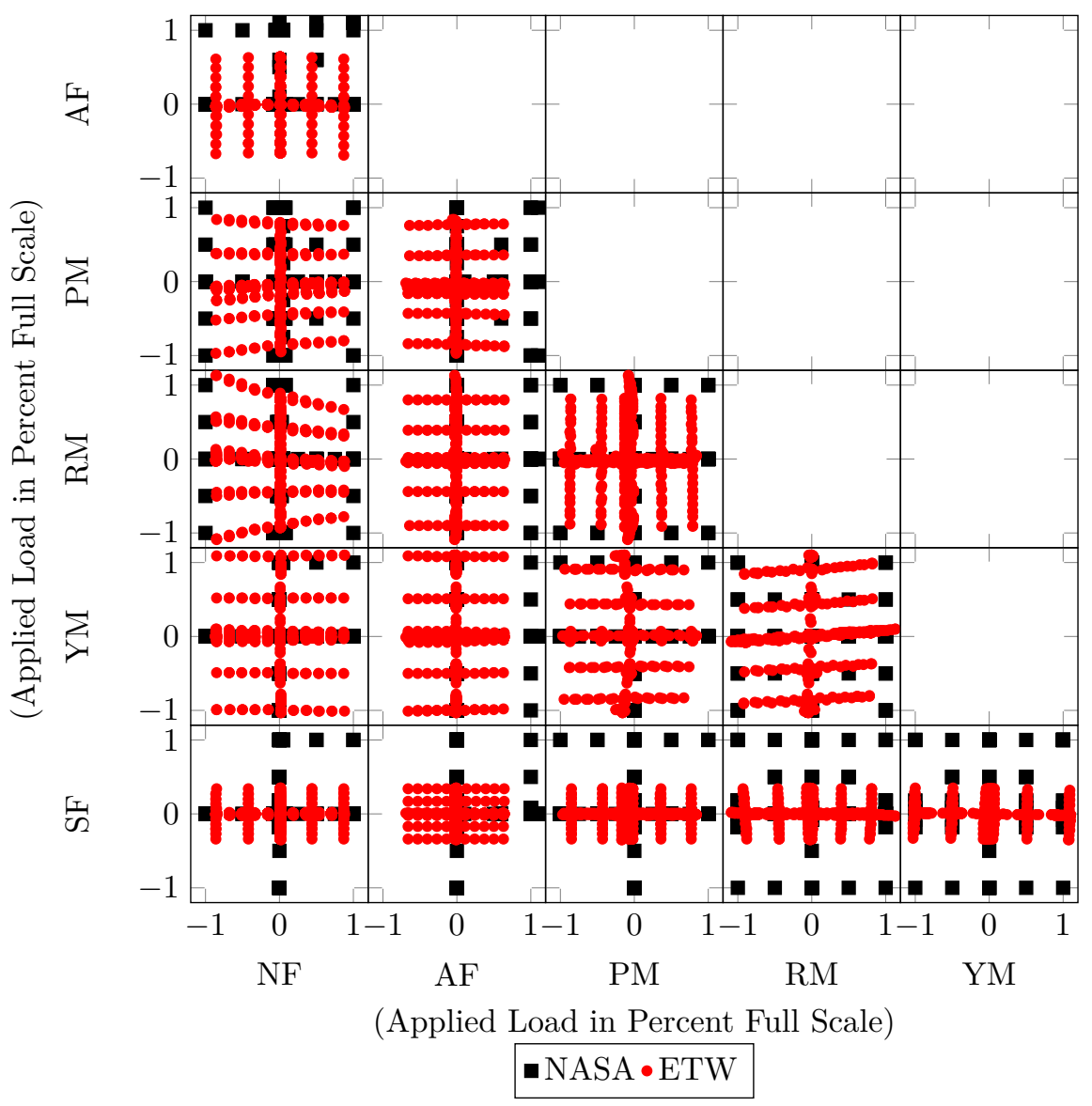

Figure 1. NASA and ETW calibration designs.

Load schedules for both calibrations are shown in Figure 1, where the red dots are ETW points, and black squares are NASA points. From the figure it is seen that AF and SF are at significantly lower load ranges, where the ETW SF full scale is $35 \%$ of NASA full scale. Some analysis issues do arise due to the large difference in applied loads between the systems. Since balances are typically modeled with a quadratic mathematical model, changes in full scales can lead to different estimates for calibration coefficients. These different estimates are due to estimating a model while on a different segment of a curve, and forcing the 
ETW data to extrapolate. Due to this large difference in full scale SF it will be ignored for the remainder of the paper. NF, AF, and PM are the primary focus of this study since these generally are the most important components during wind-tunnel testing of transport aircraft.

It is important to note that the two calibration methodologies are quite similar, both feature single-, two-, and three-component loads. This similarity in applied loads helps the two calibrations match, since they are based on similar active components. Additionally, the NASA load schedule is not symmetrical as shown by the AF component where no negative AF is applied.

\section{B. Room Temperature Calibration Analysis}

Calibration coefficients for each of the calibrations in percent of full-scale effects (F.S.E.) are in Table 1. F.S.E. is used to help illustrate contributions of each term to the full scale output of the corresponding component. For example, the NF interaction term for AF is $15.4 \%$, and signifies that when full scale normal is applied $15.4 \% \mathrm{AF}$ full scale output is expected. Balances are designed to minimize interactions between components in an effort to provide independent, linear, responses.

Table 1. NASA and ETW room temperature calibration coefficients, \% full scale effect.

\begin{tabular}{|c|c|c|c|c|c|c|c|c|c|c|c|c|}
\hline \multirow[b]{2}{*}{ Term } & \multicolumn{6}{|c|}{ NASA Calibration } & \multicolumn{6}{|c|}{ ETW Calibration } \\
\hline & NF & $\mathrm{AF}$ & $\mathrm{PM}$ & $\mathrm{RM}$ & YM & $\mathrm{SF}$ & NF & $\mathrm{AF}$ & $\mathrm{PM}$ & $\mathrm{RM}$ & YM & $\mathrm{SF}$ \\
\hline $\mathrm{NF}$ & 0.00 & 15.40 & 0.47 & 0.14 & -0.32 & -1.41 & 0.00 & 14.80 & 0.20 & 1.00 & -0.37 & -1.72 \\
\hline $\mathrm{AF}$ & -0.20 & 0.00 & -0.02 & 0.00 & -0.02 & 0.05 & -0.18 & 0.00 & -0.29 & -0.03 & -0.14 & 0.07 \\
\hline $\mathrm{PM}$ & 0.21 & -1.63 & 0.00 & -0.43 & 0.11 & -0.54 & 0.19 & -1.62 & 0.00 & -0.59 & 0.04 & -0.59 \\
\hline $\mathrm{RM}$ & -0.82 & -0.15 & 0.40 & 0.00 & 2.06 & 0.17 & -0.83 & -0.15 & 0.36 & 0.00 & 2.17 & 0.59 \\
\hline YM & 0.05 & 0.94 & -0.17 & 23.30 & 0.00 & 0.08 & 0.05 & 0.89 & -0.15 & 23.10 & 0.00 & 0.09 \\
\hline $\mathrm{SF}$ & -0.08 & -0.20 & -0.03 & -5.18 & 0.05 & 0.00 & -0.07 & -0.22 & -0.03 & -5.05 & 07 & 0.00 \\
\hline $\mathrm{NF}^{*} \mathrm{NF}$ & 0.10 & -5.67 & 0.05 & -0.02 & -0.01 & 0.29 & 0.07 & -5.82 & 0.16 & 0.21 & 0.00 & 0.25 \\
\hline $\mathrm{NF}^{*} \mathrm{AF}$ & 0.04 & 1.94 & 0.03 & 0.02 & -0.01 & 0.14 & -0.02 & 2.04 & -0.31 & 0.00 & .01 & 0.02 \\
\hline $\mathrm{NF}^{*} \mathrm{PM}$ & 0.00 & 5.02 & 0.12 & 0.05 & 0.01 & -0.06 & 0.00 & 5.17 & 0.11 & 0.01 & 0.05 & -0.19 \\
\hline $\mathrm{NF}^{*} \mathrm{RM}$ & 0.00 & 0.08 & 0.01 & -0.08 & 1.34 & 4.60 & -0.01 & 0.05 & -0.01 & 0.03 & 1.24 & 8.06 \\
\hline $\mathrm{NF}^{*} \mathrm{YM}$ & -0.01 & -0.05 & -0.05 & 0.43 & -0.17 & 0.08 & 0.01 & -0.09 & -0.03 & 0.39 & -0.35 & 0.01 \\
\hline $\mathrm{NF}^{*} \mathrm{SF}$ & 0.02 & 0.00 & -0.01 & -0.29 & 0.03 & -0.30 & 0.03 & 0.00 & 0.00 & -0.19 & 0.30 & 0.57 \\
\hline $\mathrm{AF}^{*} \mathrm{AF}$ & 0.02 & 0.08 & 0.05 & -0.03 & 0.00 & -0.13 & -0.01 & 0.04 & 0.05 & 0.10 & -0.03 & -0.17 \\
\hline $\mathrm{AF}^{*} \mathrm{PM}$ & -0.07 & -0.20 & -0.12 & 0.00 & -0.02 & 0.01 & -0.06 & -0.21 & -0.11 & 0.00 & 0.00 & 0.01 \\
\hline $\mathrm{AF}^{*} \mathrm{RM}$ & -0.01 & 0.00 & 0.00 & & -0.01 & 0.41 & 0.00 & 0.02 & -0.01 & 0.00 & 0.02 & 04 \\
\hline $\mathrm{AF}^{*} \mathrm{YM}$ & 0.01 & 0.02 & -0.01 & -0.02 & -0.06 & -0.29 & 0.00 & -0.03 & 0.00 & 0.03 & -0.02 & -0.29 \\
\hline $\mathrm{AF}^{*} \mathrm{SF}$ & 0.00 & 0.00 & -0.01 & & & & & & & -0.05 & & -0.01 \\
\hline $\mathrm{PM}^{*} \mathrm{PM}$ & 0.04 & 2.19 & 0.00 & -0.05 & -0.02 & 0. & 0.03 & 2.21 & 0.03 & -0.07 & -0.04 & 0.10 \\
\hline $\mathrm{PM}^{*} \mathrm{RM}$ & 0.00 & -0.02 & 0.00 & & & & & & 03 & & 38 & -0.04 \\
\hline $\mathrm{PM}^{*} \mathrm{YM}$ & 0.00 & 0.00 & 0.00 & -0.38 & -0.03 & -0.07 & -0.01 & 0.12 & 0.03 & -0.53 & 0.15 & -0.34 \\
\hline $\mathrm{PM}^{*} \mathrm{SF}$ & -0.01 & 0.00 & 0.02 & 0.28 & -0.02 & 0.15 & 0.01 & 0.01 & 0.03 & 0.31 & 0.06 & 0.67 \\
\hline $\mathrm{RM}^{*} \mathrm{RM}$ & -0.04 & 3.12 & -0.03 & -0.03 & -0.05 & -0.02 & -0.02 & 3.10 & -0.03 & 0.00 & 0.00 & 0.03 \\
\hline $\mathrm{RM}^{*} \mathrm{YM}$ & -0.02 & -3.45 & -0.24 & 0.00 & -0.01 & -0.03 & -0.07 & -3.43 & -0.27 & 0.00 & 0.04 & -0.01 \\
\hline $\mathrm{RM}^{*} \mathrm{SF}$ & -0.08 & -0.11 & -0.10 & 0.00 & 0.00 & 0.00 & -0.09 & -0.10 & -0.08 & 0.03 & 0.01 & 0.02 \\
\hline $\mathrm{YM}^{*} \mathrm{YM}$ & 0.01 & -0.12 & 0.02 & -0.01 & -0.02 & 0.02 & 0.03 & -0.08 & 0.01 & -0.04 & 0.01 & -0.03 \\
\hline $\mathrm{YM}^{*} \mathrm{SF}$ & 0.00 & 0.26 & 0.00 & 0.02 & -0.01 & 0.02 & 0.01 & 0.28 & 0.01 & 0.00 & 0.02 & -0.06 \\
\hline $\mathrm{SF}^{*} \mathrm{SF}$ & 0.00 & -0.05 & 0.00 & 0.00 & 0.00 & 0.01 & 0.00 & 0.02 & 0.00 & -0.03 & 0.01 & 0.01 \\
\hline
\end{tabular}

Discrepancies between the two matrices are illustrated as a bar chart in Figure 2; for brevity only NF, $\mathrm{AF}$, and PM are shown. These discrepancies display the magnitude of difference between expected outputs from each calibration, such as a $0.55 \%$ discrepancies between AF estimates when full-scale NF is applied. Other high discrepancies between that calibrations are NF and AF interaction on PM. Despite the near $0.5 \%$ discrepancies, this does not suggest that either of the two calibration systems or methodologies are wrong, simply that there might be differences in how the balance is referenced or leveled.

Observed differences in the linear iteration portion of the matrices can be due to angular of positional offsets. For example, if the balance is placed at different pitch angles, it would be seen as a change in the NF interaction on AF. Similarly, a difference in roll angle would yield a change in the NF interaction on SF. 
These rotational difference would also change the interactions of the NF component, but not as significantly as $\mathrm{AF}$ and SF due to their increased sensitivity. Positional differences, tend to affect changes in the moment components, such as an x-displacement influencing a change in the NF interaction on PM.
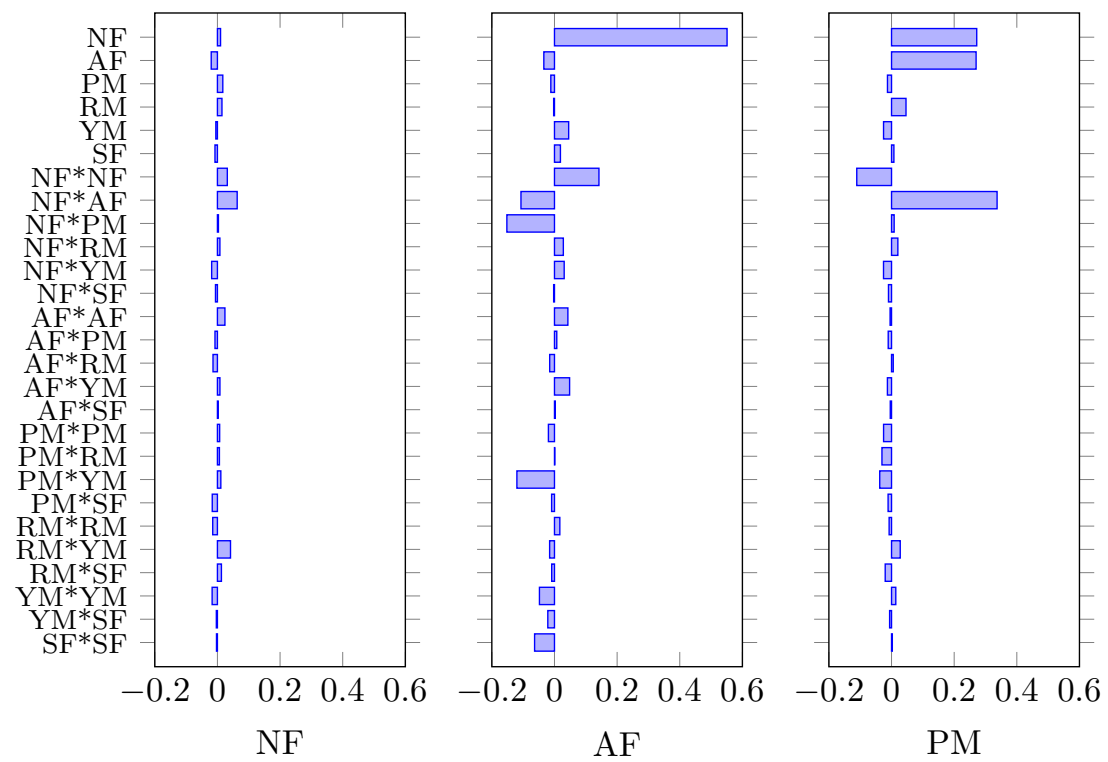

(Percent Difference of Full Scale Effect)

Figure 2. Room temperature differences between NASA and ETW calibrations.

\section{Balance Coordinate Analysis}

The effects of the discrepancies seen in Figure 2 can also be illustrated by calculating loads via both matrices from a common voltage. In the case shown in Figure 3, the ETW matrix is used to convert ideal loads to a common voltage afterwards the NASA matrix is used to recompute loads. Differences in the re-estimated loads are caused by the differences in the calibration matrices, where if the matrices were identical, no differences would be found. Ideal loads in this case were based on a rotatable central composite design to eliminate influences from either the NASA or ETW calibration design. Figure 3 shows, in black, the residual errors between the ideal loads and the back computed loads through the two matrices. In an ideal case, the differences would be minimal as shown by the NF component in this figure, where the discrepancies are within $\pm 0.05 \%$ as expected by the results of Figure 2 indicating NF agreement.

Several trends are seen in the load discrepancies, such as a strong correlation between applied NF and AF discrepancies. Other notable trends are applied NF and AF on the PM discrepancies. These trends are related to the matrix discrepancies found in Figure 2 such as NF interaction on AF. As discussed prior, one possible source for trends in the load discrepancies are differences in the position and angle reference used for both calibration systems. An optimization routine was implemented to find possible rotations and translations of the balance coordinate system. ${ }^{6}$ This method finds a rotation matrix and coordinate translation that minimizes the load discrepancies shown in Figure 3. Estimated offsets that partially correlate the two calibration matrices are shown in Table 2.

Table 2. Estimated angle and position offsets.

\begin{tabular}{cc|cc}
\hline \hline Angle & QA & \multicolumn{2}{c}{ Position } \\
Offset (Deg.) & (Deg.) & \multicolumn{2}{|c}{ Offset (in) } \\
\hline Roll -0.019 & -0.012 & $\mathrm{x}$ & 0.005 \\
Pitch -0.036 & -0.007 & $\mathrm{y}$ & 0.011 \\
Yaw -0.011 & 0.003 & $\mathrm{z}$ & -0.019 \\
\hline \hline
\end{tabular}




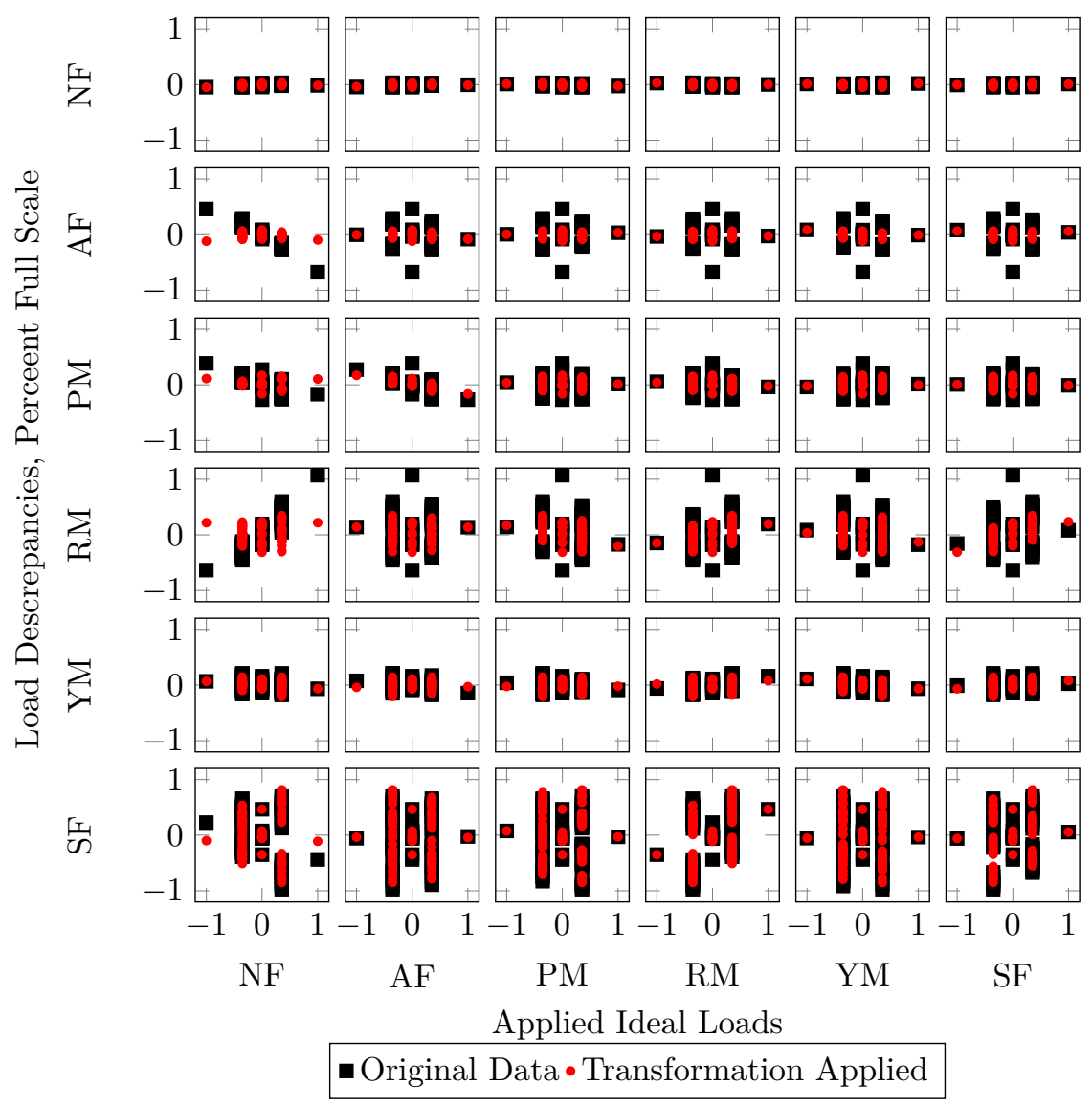

Figure 3. Load discrepancies estimated by NASA and ETW matrices.

Quality assurance (QA) measurements of the ETW load adapter for the NTF-118A are also listed in Table 2. Comparing estimated offsets to QA measurements, the two sets of values only agree for the roll measurements. Pitch QA value only accounts for $20 \%$ of the estimated total offset, which suggest that other hardware may affect the total offset between the systems. Presented here are only the QA measurements of one part of one system, not total aggregate offset between the angle references of the NASA calibration system and the ETW BCM. These offset estimates can help guide further QA measurements to fully understand differences between calibration systems.

Applying the estimated offsets through a rotation matrix and coordinate translation to one set of loads yields the red points in Figure 3. This shows a large reduction in the residual errors for the AF, PM, and RM components. It should be noted that a trend still exists between the PM residual and AF, as shown by the downward trend. This trend may be due to a z-offset, but the optimization routine did not indicate that this trend was supported by the other components that are affected by z-offsets. For example, the z-offset required to reduce the AF on PM trend would lead to higher RM load discrepancies due to SF.

A new calibration matrix was estimated using the modified data, that accounts for the shifts seen in Table 2. Shown in Figure 4 are the discrepancies between the two calibrations, where one matrix is adjusted. Comparing this figure to Figure 2, the NF interactions on AF and PM components are no longer present due to the offset corrections. As mentioned prior, the AF interaction on PM still remains and was not corrected by the process. These results suggest that is it possible that angular and position offsets of the balance coordinate system are different for each of the calibration systems, which is expected. The offsets that were found are nothing alarming and are within reasonable limits of manufacturing errors as shown by the roll QA value. 

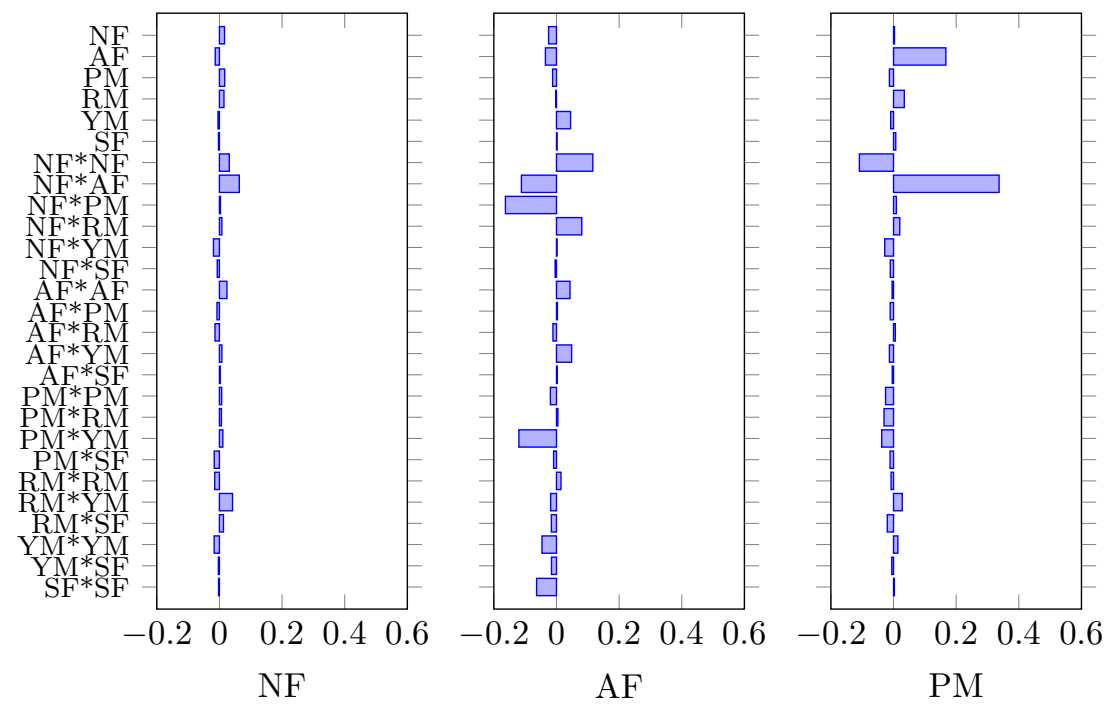

(Percent Difference of Full Scale Effect)

Figure 4. Room temperature differences between NASA and transformed ETW calibrations.

\section{Conclusion}

Comparison of the two room temperature calibrations from NASA and ETW showed that they correlate well for normal force, but have significant differences in several of the other components. Due to the differences in applied full scale loads, caution is applied to some of the analysis such as ignoring side force results.

Several discrepancies found between the two calibration matrices were shown to be related to coordinate system rotations and translations. The estimated rotational and translational offsets, significantly decreased the discrepancies found in the axial force, pitch and rolling moment components. Offset estimates were not fully correlated to quality assurance measurements of the adapter plate for the balance, but was found to closely match in roll. This lack of correlation does not disprove the results since only one part was examined and not the full offset stack-up between the two machines. These results show that it is important to build up QA measurements of as many components of the calibration system to help diagnose discrepancies between calibration systems.

Prior to applying corrections, rolling moment and axial force load discrepancies approached $1.0 \%$ and $0.5 \%$, respectively. These load discrepancies drop significantly after the application of the offset estimates to one of the calibration matrix, which suggest that the estimated offsets account for the original discrepancies found between the matrices. With the methodology shown in this paper, good agreement between the two systems is found after angular and positional corrections are applied.

\section{References}

\footnotetext{
${ }^{1}$ Quest, J. and Schimanski, D., "Tools \& Techniques for High Reynolds Number Testing - Status \& Recent Improvements at ETW," Aerospace Sciences Meetings, American Institute of Aeronautics and Astronautics, Jan. 2003.

${ }^{2}$ Rivers, M. and Dittberner, A., "Experimental Investigations of the NASA Common Research Model (Invited)," Fluid Dynamics and Co-located Conferences, American Institute of Aeronautics and Astronautics, June 2010.

${ }^{3}$ Hufnagel, K. and Quade, M., "The 2nd Generation Balance Calibration Machine of Darmstadt University of Technology (TUD)," Aerospace Sciences Meetings, American Institute of Aeronautics and Astronautics, Jan. 2007.

${ }^{4}$ Rhew, R. D. and Parker, P. A., "Wind Tunnel Balance Calibration: Are 1,000,000 Data Points Enough?" 10th International Symposium on Strain-Gage Balances, May 2016.

${ }^{5}$ Burns, D. E., Toro, K. G., Parker, P. A., Rivers, M. S., Quin, H., and Wright, M., "The Evaluation of Cryogenic Force Balance Calibration Methodologies with Respect to Wind Tunnel Results," 11th International Symposium on Strain-Gauge Balances, Cologne, Germany, May 2018.

${ }^{6}$ Toro, K. and Parker, P. A., "Impact and Estimation of Balance Coordinate System Rotations and Translations in WindTunnel Testing," AIAA AVIATION Forum, American Institute of Aeronautics and Astronautics, June 2017.
} 